\title{
La relación inversa entre ejercicio y enfermedad coronaria es dosis dependiente
}

Exercise Type and Intensity in Relation to Coronary Heart Disease in Men. Tanasescu M, Leitzmann M, Rimm E y col. JAMA 2002;288:19942000

\section{Objetivo}

Evaluar la relación entre la cantidad, intensidad y tipo de la Actividad Física (AF), y el riesgo de enfermedad coronaria (EC) en hombres.

\section{Diseño}

Estudio prospectivo de una cohorte de hombres, que respondieron un detallado cuestionario salud enviado en 1986 y re-enviado cada 2 años hasta 1998.

\section{Lugar}

Escuela de Salud Pública y de Medicina de Harvard y el Departamento de Medicina del Brigham and Women's Hospital, de Estados Unidos.

\section{Población}

Se evaluó una cohorte de 44452 hombres enrolados en el Estudio de Seguimiento de Profesionales de la Salud de Estados Unidos, entre 40 y 75 años, que respondieron al cuestionario y que fueron seguidos en forma prospectiva.

Evaluación de los factores pronósticos y resultados principales Se registraron factores de riesgo potenciales de EC, nuevos diagnósticos de EC, y AF recreativa.Se cuantificó en forma detallada la AF consignándola como el tiempo promedio semanal de cada actividad.Se realizó una categorización a la AF en quintilos de intensidad según los equivalentes de trabajo metabólico (METs).El entrenamiento con pesas comenzó a registrarse a partir de 1990. La caminata física fue dividida en tres categorías de acuerdo con la ve- locidad del paso.El resultado primario evaluado fue nuevo infarto de miocardio o enfermedad coronaria fatal.

\section{Resultados principales}

Los hombres más activos tendieron a tener:un menor índice de masa corporal;a ser consumidores de menos cantidad de grasas totales y saturadas, mayor cantidad de fibra y alcohol, mayor cantidad de suplementos con vitamina E;y a tener menor prevalencia de tabaquismo e hipertensión.

Durante el seguimiento de 475755 personas/año ocurrieron 1700 nuevos eventos cardiovasculares. Ajustado por edad, tabaquismo y otros factores de riesgo, el riesgo relativo $(R R)$ de eventos disminuyó progresivamente desde el quintilo de menor actividad total hacia el de mayor actividad.EI RR comparando los quintilos extremos fue de:0,70 (IC 95\% 0,59-0,82). Analizando la intensidad del ejercicio la reducción del RR para el de alta intensidad fue de 0,83 (IC $95 \% 0,72-0,97$ ) y de 0,94 para el ejercicio moderado (IC 95\% 0,83$1,03)$ comparándolos con el ejercicio de baja intensidad con un valor de $p$ para la tendencia $=0,02$. El ejercicio de resistencia con pesas o máquinas (según se realizaba más de 30 minutos semanales o menos), demostró una disminución de RR del 17\% para los de menos de 30 minutos y de $35 \%$ para los de mas de 30 minutos $(p<0.001)$.Si bien el volumen total de caminata disminuyó el RR, la mayor reducción se obtuvo en los caminadores de paso intenso.

\section{Conclusiones}

El ejercicio tuvo una relación inversamente proporcional, dosis dependiente;con la aparición de enfermedad coronaria.

\section{Comentario}

Los dos aspectos más novedosos del presente trabajo son la reducción del RR de eventos coronarios por el entrenamiento de resistencia y la importancia de la intensidad de la caminata más allá del volumen.

Asimismo, confirma algunos aspectos de la AF ya postulados: la estrecha relación del sedentarismo con la mortalidad cardiovascular, y la utilidad del la capacidad funcional como predictora de eventos cardiovasculares Los resultados del estudio analizado permiten despejar dudas sobre el entrenamiento de fuerza resistencia (beneficioso desde todo punto de vista);la intensidad de la actividad (a mayor intensidad mayor beneficio); el volumen de la AF (no encontrándose que este sea el parámetro clave de la prescripción); y la intensidad del paso de la caminata (más importante que el volumen de las caminatas). No se pudieron obtener conclusiones sobre el ciclismo y la natación ya que fue muy escasa la cantidad de sujetos de la cohorte que practicaban estas disciplinas.

Conclusiones del comentador: Los beneficios asociados con la actividad física son indiscutibles. En nuestro consultorio debemos estimular la práctica de la misma, conociendo la escasa cantidad de situaciones que la contraindican y que casi todos los tipos son útiles. Nuestras metas, como profesionales de la salud, deben ser la adherencia a largo plazo y lograr que el cambio de estilo de vida sea permanente. Debemos estimular a hacer lo que al paciente le gusta hacer; no proponer metas inalcanzables ni modelos ideales; orientar a la persona que nunca hizo ejercicio a encontrar la actividad que lo satisfaga. Por último, el hábito deportivo que nace en la niñez perdura en el tiempo, es muy difícil iniciar en el deporte a un adulto que nunca realizó ejercicio, por eso la prescripción debe ser lo más temprana posible. 